\title{
Co-designing a mHealth intervention to prevent smoking relapse after birth
}

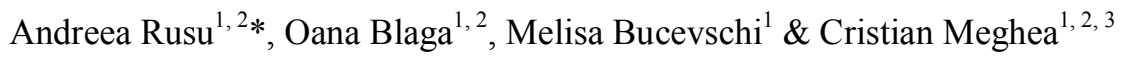 \\ ${ }^{1}$ Center for Health Policy and Public Health, Babeș-Bolyai University, Cluj-Napoca, Romania. \\ ${ }^{2}$ Department of Public Health, Babeș-Bolyai University, Cluj-Napoca, Romania. \\ ${ }^{3}$ Department of Obstetrics, Gynecology and Reproductive Biology, College of Human Medicine, Michigan State University,
}

USA.

Received 8.05.2020; Received revised 23.06.2020; Accepted 28.06.2020;

Available online 30.06.2020

\begin{abstract}
Background: Smoking during pregnancy is a well-known risk factor for both the mother and the baby. mHealth offers novel ways to implement smoking-cessation interventions with broader reach and the potential to be more successful.

Methods: We conducted twelve semi-structured interviews in November-December 2017 with women who just gave birth and quit smoking before or during pregnancy. A thematic analysis was conducted to explore women's views on the two components.

Results: Most women found the text messages easy to understand and virtually all women rated their content as relevant for preventing smoking relapse. Also, they expressed interest in using the proposed relapse prevention app and, in general, appreciated the app positively. Some women, however, felt that the smoking relapse decision is highly personal and cannot be influenced by text messages or other types of assistance programs.

Conclusions: Stay Quit Together is, to our knowledge, the first postpartum tobacco smoking relapse prevention program combining an app and text messages. This study suggests that Stay Quit Together would be accepted and used postpartum by women who spontaneously quit smoking during or before pregnancy.
\end{abstract}

Keywords: postpartum, smoking cessation, Romania, middle-income countries, mHealth.

Address of correspondence: Andreea Rusu, Center for Health Policy and Public Health, College of Political, Administrative and Communication Sciences, Babeș-Bolyai University, Str. Gen Traian Mosoiu 71, Cluj-Napoca, Romania.

E-mail: andreea.hostina@publichealth.ro

\section{Introduction}

Smoking during and around pregnancy is a major risk factor for maternal and child adverse outcomes (Keegan, Parva, Finnegan, Gerson, \& Belden, 2010). In Romania, studies show that up to $30 \%$ of women used to smoke 6 months before becoming pregnant, with half of them quitting smoking upon finding out about the pregnancy (Blaga, Brînzaniuc, Rus, Cherecheş, \& Wallis, 2017). However, many studies show that up to $70 \%$ of those who quit smoking during pregnancy relapse after giving birth, many of them in the first three months (Winickoff et al., 2010). Currently there are very few national programs in Romania and many other low and middle-income countries (LMIC) that specifically address pregnancy smoking cessation and postpartum relapse prevention and the only efforts that are being made are at a research-level, targeting low numbers of participants. Tailored intervention targeting postpartum smoking relapse prevention are needed to overcome this deficit in Romania and other LMIC.

In the postpartum period, smoking relapse poses greater health risk for the mother ("Women \& Tobacco," 2011) and the infant, including infections, sudden infant death syndrome, middle ear disease, reduced lung function, respiratory illnesses, delays in cognitive development, and cancer (Hofhuis, 2003). As such, prevention of postpartum smoking relapse has positive implications for both the mother and the baby.

Mobile health interventions have a large potential to improve health and reduce diseases (Beratarrechea et al., 2014). Yet, despite the serious adverse outcomes of smoking, there are only few mobile health programs tailored for pregnant or postpartum women, most with low 
enrollment and unknown effectiveness (Heminger, Schindler-Ruwisch, \& Abroms, 2016).

One of the best strategies to boost enrollment rates and improve acceptability, retention, and effectiveness of mHealth interventions is to involve the participants in the co-design of the interventions (Kumar et al., 2013).

To add to the evidence on the usability of mHealth interventions tailored to the needs of postpartum women, we conducted interviews with women who just gave birth and quit smoking before or during pregnancy. We explored women's views and suggested changes related to the design, content or personalization of the Stay Quit Together smoking relapse prevention program consisting of the iCoach smoking cessation mobile app and a short message service (SMS) component, tailored to the needs of postpartum women and their partners.

The aim of this paper is to explore the views of postpartum women on the two components of the Stay Quit Together postpartum smoking relapse prevention intervention - the iCoach mobile application and the text messages - in order to co-design Stay Quit Together and inform future research on theory-grounded mHealth interventions delivered through mobile devices.

\section{Method}

\section{The intervention}

The intervention consists of two components: the modified iCoach app and a short message service (SMS) component. The iCoach app was developed under a tender from the European Commission, and translated in $23 \mathrm{EU}$ languages, including Romanian. The application was available free of charge, both online and as a mobile app. For this intervention, the iCoach app was adapted and is made available to users through personalized links. The app contains three main sections: Daily tips (around 630 items), Library (around 120 items) and Panic tips (around 100 items). The "Daily tips" section consists of advice that the user receives every day to maintain motivation to stay smoke-free, while the "Library" section offers additional information about smoking for the users that are interested in further reading.

The five stages that the app was based on were: (1) I do not plan on stopping, (2) I should stop, but I am still in doubt, (3) I will stop smoking shortly, (4) I have just stopped, (5) I have stopped for some time now. The app is tailored to each person because when first using the app, the user fills in a short questionnaire in order to set goals and adapt the information to what he or she needs.

We developed the SMS component consisting of 350 text messages for postpartum women and 230 messages for their life partners informed by our prior work (Meghea et al., 2018) grounded in the Motivational and Problem Solving framework (Reitzel et al., 2010) and with a focus on enhancing dyadic efficacy for smoking cessation (Sterba et al., 2011). The SMSs fall into four categories: general health and well-being, motivation enhancement, confidence enhancement, and coping enhancement. Among the messages, there are cases when women are asked to answer with a number from a scale or a keyword.

\section{Setting}

The formative qualitative data was collected during the clinic birth stay from mothers who recently gave birth at the two largest state-owned obstetrics- gynaecology clinics in Cluj-Napoca, Romania - Obstetrics- Gynaecology 1
Clinic (5 interviews) and Obstetrics-Gynaecology 2 "Dominic Stanca" Clinic (7 interviews).

\section{Recruitment and screening procedures}

A convenience sample of women were interviewed by a trained data collector, who explained the objectives and the procedures of the study and invited women to participate in the study provided they sign an informed consent. The eligibility criteria were: 18 years of age or older, just gave birth, married or having a stable partner, quit smoking before or during pregnancy and willing to sign the study's informed consent. The average age of the women was 28.25 years $(\mathrm{SD}=3.16$, range $24-35)$. Ethical approval was obtained from the Ethics Committee of the Babeș-Bolyai University (\#36329/27.10.2017). Women who accepted to participate in the study were offered a digital baby thermometer as an incentive.

\section{Data collection}

The total number of women who went through the eligibility process was 76 , but only 12 of them were eligible and interested to participate. All eligible women who expressed interest in participating and filled in the consent form were interviewed by a female graduate student. Between November and December 2017 we conducted twelve semi-structured interviews based on a pre-set interview guide, with women who just gave birth and quit smoking before or during pregnancy. Ten interviews were conducted face-to-face in the clinics and two were conducted via telephone, due to women's unavailability to participate in the interview in the clinic. The phone interviews were conducted after the women received by e-mail the same screenshots and information as the women in the clinics. The interviews lasted between 10 and 30 minutes ( $\mathrm{SD}=6.74,11: 20$ minutes $-28: 51$ minutes).

\section{The interview guide}

The semi-structured interview guide consisted of two parts. The first part of the interview guide covered the following topics: questions on socio-demographics, smoking narratives, experience with smoking and smoking cessation (including smoking history and quitting attempts), commitment to remain smoke free, relationship with the partner/husband (Reitzel et al., 2010), including types of interactions regarding smoking, teamwork standards, dyadic efficacy, and coping (Sterba et al., 2011), and support for smoking cessation. The second part of the interview elicited information about the iCoach mobile application and the text messages. For the app, women were shown screenshots of the application and were asked to state their opinion on different aspects. We were interested in the willingness of women to download the application, likes and dislikes regarding the application, and their opinion regarding the most useful section of the application. In terms of the SMSs, we assessed how many SMSs they preferred to receive per day, when to receive them, and the degree of personalization. The participants were shown samples of SMSs and answered specific questions about the level of understanding, relevance, level of motivation, the presence of offensive words etc.

\section{Data analysis}

Eleven of the twelve interviews were audio-recorded, including the two interviews via telephone, with the permission of the participants, and transcribed verbatim for analysis. Just one of the twelve women did not give her permission to audio-record the interview so notes were 
taken during the interview. Transcripts were analysed (Braun \& Clarke, 2006) using MaxQDA (VERBI Software - Consult - Sozialforschung $\mathrm{GmbH}$ ). The analysis involved developing a coding scheme, coding the data, identifying the themes and analysing them. Data was coded separately by two research assistants and after a first round of coding the codes were reorganized and grouped into categories, inter-coder reliability being assessed periodically. The final coding system was developed through a consensus process, discrepancies in coding being solved through face-to-face discussions between the coders.

\section{Results}

Six of the women stated that they started smoking before the age of 20, two said they started in their twenties and for the remaining four we don't have this information. Regarding the quit attempts, four of the women said they quit during previous pregnancies and five said they tried to quit before but it only lasted for short periods of time. The remaining three women didn't answer this question. Eight of the partners were smokers and four non-smokers. Nine of the interviewed women live in an urban area, one of them lives in a rural area and for the remaining two we don't have any data regarding their residence.

Three main relevant themes were identified: a) opinions about the overall intervention, b) about the mobile application and c) about the text messages. Participants were shown screenshots of the application's content and example SMSs.

\section{Women's opinion about the overall intervention}

Nine of the 12 women said that they would accept to download the application on their mobile devices and that their opinion about the implementation of a program designed to prevent smoking relapse after birth is a positive one, although some of them were not quite sure about its efficacy.

"I would download the application even if I know it wouldn't help me very much" (Subject 104, 29 years old, living in urban area)

"Yes, yes, yes. I have used [other apps], before I quit smoking I've searched for all kinds of books, I've read, I've studied. I was quite interested even before, so that I know what I need to do [...]" (Subject 101, 32 years old, living in urban area)

Only one of the interviewed women said that she would not be interested in the intervention:

"No. This is something that people have to decide for themselves. They have to be ambitious. [...] No, I don't feel the need, I don't know..." (Subject 102, 28 years old, unknown living area)

Most of the women stated that no concerns arise regarding the intervention. The only concern that emerged during the interviews was related to the program's safety and data usage:

"The safety of the program - the personal data, so nobody can access it" (Subject 109, 27 years old, living in urban area)

Women's opinion about the mobile application

Out of the 12 women, nine said they like the application, the only comment being related to the appearance:
"It seems interesting, it really seems interesting, I haven't seen anything that I don't like". (Subject 105, 31 years old, living in rural area)

"[...] it's simple. And I think it's helpful for those who want to quit" (Subject 106, 31 years old, living in urban area)

"There is too much text. There should be more images" (Subject 104, 29 years old, living in urban area)

When asked what section they find the most useful, most of the women pointed to the Panic Advices and the Daily Advices:

"The one with the panic" (Subject 105, 31 years old, living in rural area)

"There is something there with some suggestions, with 'I can quit smoking, I want, but I can't' " (Subject 103, unknown age, living in urban area)

After seeing the application, women were asked if they would use the application to remain smoke-free. Five of the women said that they would probably find it helpful and two of them said they would not use it. The remaining five women didn't answer the question.

"Yes, I think it would help me" (Subject 109, 27 years old, living in urban area)

"Yes, it would be a small added aid" (Subject 110, 36 years old, living in urban area)

\section{Women's opinion about the text messages}

The participants were shown samples of the messages that were going to be sent to women in the first 6 weeks after birth. Similar messages will be sent to their life partners. We asked them to evaluate the content regarding the motivation, relevance, addressing formulas and whether they would rather receive personalized SMSs. Furthermore, women were asked if they would read the messages, if they would take the time to answer them when asked to, what would be the best time to receive them and how often.

Two of the women said they would not always read the messages:

"Not always, I honestly think that not always [I would read the SMSs]." (Subject 102, 28 years old, unknown living area)

On the other hand, eight women stated that they would read the SMSs:

"Of course I would read it all, yes. Well, I read everything I receive because I don't know what it's about" (Subject 101, 32 years old, living in urban area)

"Probably, when I'll have the time" (Subject 108, 24 years old, living in urban area)

"Yes, I don't think they are that long so that I don't have the time" (Subject 109, 27 years old, living in urban area)

In some instances, women would receive messages that ask them to evaluate their motivation or the importance of staying smoke-free on a scale from 1 to 10 , where 1 is the lowest and 10 is the highest level. They should send this number as a reply to the message, so we asked the women what their opinion was regarding this aspect. Five of the women stated that they would reply with an answer, two of the women said that they would not agree to answer the SMSs and the remaining five didn't answer the question:

"Yes, I would like to interact with the message I receive" (Subject 109, 27 years old, living in urban area)

"No, this thing with sending back never tempted me" (Subject 105, 31 years old, living in rural area) 
We also asked women about their view upon the SMS frequency; we suggested an average of three messages per day, five days per week, for 6 weeks. The majority said that this would be fine and they wouldn't mind. However, there were women who said the number should be lower, even stating that "one is enough".

"Yes, it would be OK. I wouldn't mind at all" (Subject 104, 29 years old, living in urban area)

"From my point of view, no [I wouldn't mind], but people are different. I'm sure there are people who would mind." (Subject 101, 32 years old, living in urban area)

All of the interviewed women said that the messages were easy to understand and none of the words were found offensive. Also, 11 of the 12 women rated the relevance of the SMSs 6 or higher on a scale from 1 to 10 . Furthermore, on the same scale, 9 of the women stated that the motivation that the messages bring is above 5 . The other three women didn't think that the messages raised their motivation.

\section{Discussion}

The present Stay Quit Together is, to our knowledge, the first postpartum tobacco smoking relapse prevention program combining an app and text messages. This study provides new insights on the potential usability and acceptability of a co-designed postpartum smoking relapse prevention intervention which includes a mobile app and text messages in a middle-income Eastern European country. In line with previous research, this study found that mobile technology can be useful for smoking-related interventions (Munafo, 2017). Consistent with other studies (Abroms et al., 2015), we found that potential participants would accept to use such an intervention without major concerns and that the intervention is likely to help with tobacco relapse prevention postpartum. The present study contributes to the existing body of knowledge by filling a gap, particularly in LMIC, in the process of finding the appropriate tools to deliver a theorybased intervention through digital means and the need to localize and personalize the intervention in terms of content, design or usability.

Women's narratives regarding the overall postpartum smoking relapse intervention suggested that the app would be well-received and the messages were highly relevant, the general feeling about the intervention was positive, but some were unsure about the potential efficacy of the program. Another concern was related to the safety of the personal data. Overall, most women said they would accept to download the application on their mobile devices and had a positive opinion about such a program designed to prevent smoking relapse after birth. Since, to our knowledge, this is the first postpartum tobacco smoking relapse prevention program combining an app and text messages, there is no direct comparison of the overall program to the few existing mHealth interventions focused on pregnancy and postpartum. These interventions consisted either of text messages or apps focused on smoking cessation and abstinence during pregnancy and after birth (Heminger et al, 2016).

In general women had a positive opinion about the app component of the Stay Quit Together program. On the text message component of Stay Quit Together, women found the text messages easy to understand, and virtually all women rated the messages as relevant for preventing smoking relapse postpartum. Some women, however, felt that the smoking relapse decision is highly personal and cannot be influenced by text messages or other types of assistance programs. Prior text message interventions focused on cessation and abstinence during and after pregnancy were rated favourably by users in terms of content and being helpful for quitting (Abroms et al., 2015). Moreover, if the participants were asked to rate their experience, most of them provided high scores (Abroms, Chiang, Macherelli, Leavitt, \& Montgomery, 2017). Other studies report that, even if it is not fully conclusive, data shows that SMS-based interventions can help increase cessation rates if they are delivered together with the usual care (Naughton et al., 2017). These prior findings are in line with ours, but the program that we propose goes more in-depth in terms of complexity, bringing the combination between two components that were previously used separately, the SMSs and the App.

\section{Conclusions}

The findings suggest that a postpartum smoking relapse prevention program combining text messages and a mobile application would be accepted and used by women who spontaneously quit smoking during or before pregnancy. Women found such an app useful and the messages highly relevant for staying smoke free. Such a program will need to clearly explain to potential participants its premise for success. The study also shows how women's' experiences and perspectives can effectively inform the development and adaptation of mHealth interventions in under-researched social environments. This paper provides new insights into postpartum smoking relapse prevention programs that include text messages and apps, from a middle-income former communist country in Eastern Europe, especially because prior interventions didn't have a combined approach.

Funding: This work was supported by a grant of Ministry of Research and Innovation, CNCS - UEFISCDI, project number PN-III-P4-ID-PCE-2016-0632, within PNCDI III. Views in this manuscript reflect those solely of the authors.

\section{Conflict of Interests}

The authors declare no conflict of interest.

\section{References}

Abroms, L. C., Chiang, S., Macherelli, L., Leavitt, L., \& Montgomery, M. (2017). Assessing the National Cancer Institute's SmokefreeMOM Text-Messaging Program for Pregnant Smokers: Pilot Randomized Trial. Journal of Medical Internet Research, 19(10), e333. https://doi.org/10.2196/jmir.8411

Abroms, L. C., Johnson, P. R., Heminger, C. L., van Alstyne, J. M., Leavitt, L. E., Schindler-Ruwisch, J. M., \& Bushar, J. A. (2015). Quit4baby: Results from a pilot test of a mobile smoking cessation program for pregnant women. JMIR MHealth and UHealth, 3(1), e10. https://doi.org/10.2196/mhealth.3846

Beratarrechea, A., Lee, A. G., Willner, J. M., Jahangir, E., Ciapponi, A., \& Rubinstein, A. (2014). The impact of mobile health interventions on chronic disease outcomes in developing countries: A systematic review. Telemedicine Journal and E-Health : The Official Journal 
of the American Telemedicine Association, 20(1), 75-82. https://doi.org/10.1089/tmj.2012.0328

Blaga, O. M., Brînzaniuc, A., Rus, I. A., Cherecheș, R. M., \& Wallis, A. B. (2017). Smoking and Smoking Cessation During Pregnancy. An Analysis of a Hospital Based Cohort of Women in Romania. Journal of Community Health, 42(2), 333-343. https://doi.org/10.1007/s10900016-0259-6

Braun, V., \& Clarke, V. (2006). Using thematic analysis in psychology. Qualitative Research in Psychology, 3(2), 77-101. https://doi.org/10.1191/1478088706qp063oa

Heminger, C. L., Schindler-Ruwisch, J. M., \& Abroms, L. C. (2016). Smoking cessation support for pregnant women: Role of mobile technology. Substance Abuse and Rehabilitation, $\quad 7, \quad 15-26$. https://doi.org/10.2147/SAR.S84239

Hofhuis, W. (2003). Adverse health effects of prenatal and postnatal tobacco smoke exposure on children. Archives of Disease in Childhood, 88(12), 1086-1090. https://doi.org/10.1136/adc.88.12.1086

Keegan, J., Parva, M., Finnegan, M., Gerson, A., \& Belden, M. (2010). Addiction in pregnancy. Journal of Addictive Diseases, 29(2),

175-191. https://doi.org/10.1080/10550881003684723

Kumar, S., Nilsen, W. J., Abernethy, A., Atienza, A., Patrick, K., Pavel, M., ... Swendeman, D. (2013). Mobile health technology evaluation: The mHealth evidence workshop. American Journal of Preventive Medicine, 45(2), 228236. https://doi.org/10.1016/j.amepre.2013.03.017

Meghea, C., Brinzaniuc, A., Sidor, A., Chereches, R., Mihu, D., Iuhas, C., ... Blaga, O. (2018). A couples-focused intervention for smoking cessation during pregnancy: The study protocol of the Quit Together pilot randomized controlled trial. Tobacco Prevention \& Cessation, 4(May) https://doi.org/10.18332/tpc/89926
Munafo, M. (2017). How Can Technology Support Smoking Cessation Interventions? Nicotine \& Tobacco Research : Official Journal of the Society for Research on Nicotine and Tobacco, 19(3), 271-272. https://doi.org/10.1093/ntr/ntx019

Naughton, F., Cooper, S., Foster, K., Emery, J., Leonardi-Bee, J., Sutton, S., . . Coleman, T. (2017). Large multi-centre pilot randomized controlled trial testing a low-cost, tailored, self-help smoking cessation text message intervention for pregnant smokers (MiQuit). Addiction (Abingdon, England), 112(7), 1238-1249. https://doi.org/10.1111/add.13802

Reitzel, L. R., Vidrine, J. I., Businelle, M. S., Kendzor, D. E., Costello, T. J., Li, Y.,... Wetter, D. W. (2010). Preventing postpartum smoking relapse among diverse low-income women: A randomized clinical trial. Nicotine \& Tobacco Research : Official Journal of the Society for Research on Nicotine and Tobacco, 12(4), 326-335. https://doi.org/10.1093/ntr/ntq001

Sterba, K. R., Rabius, V., Carpenter, M. J., Villars, P., Wiatrek, D., \& McAlister, A. (2011). Dyadic efficacy for smoking cessation: Preliminary assessment of a new instrument. Nicotine \& Tobacco Research : Official Journal of the Society for Research on Nicotine and Tobacco, 13(3), 194-201. https://doi.org/10.1093/ntr/ntq236

MAXQDA, software for qualitative data analysis [Computer software]. Berlin, Germany.

Winickoff, J. P., Healey, E. A., Regan, S., Park, E. R., Cole, C., Friebely, J., \& Rigotti, N. A. (2010). Using the postpartum hospital stay to address mothers' and fathers' smoking: The NEWS study. Pediatrics, 125(3), 518-525. https://doi.org/10.1542/peds.2009-0356

Women \& Tobacco (2011, April 14). Retrieved from http://www.therapystore.com/smoking/women tobacco.htm 